\title{
Out of sight, out of mind: Matching bias underlies confirmatory visual search
}

\author{
Jason Rajsic $^{1}$ • J. Eric T. Taylor ${ }^{1}$. Jay Pratt ${ }^{1}$
}

Published online: 20 December 2016

(C) The Psychonomic Society, Inc. 2016

\begin{abstract}
Confirmation bias has recently been reported in visual search, where observers who were given a perceptual rule to test (e.g. "Is the $p$ on a red circle?") search stimuli that could confirm the rule stimuli preferentially (Rajsic, Wilson, \& Pratt, Journal of Experimental Psychology: Human Perception and Performance, 41(5), 1353-1364, 2015). In this study, we compared the ability of concrete and abstract visual templates to guide attention using the visual confirmation bias. Experiment 1 showed that confirmatory search tendencies do not result from simple low-level priming, as they occurred when color templates were verbally communicated. Experiment 2 showed that confirmation bias did not occur when targets needed to be reported as possessing or not possessing the absence of a feature (i.e., reporting whether a target was on a nonred circle). Experiment 3 showed that confirmatory search also did not occur when search prompts referred to a set of visually heterogenous features (i.e., reporting whether a target on a colorful circle, regardless of the color). Together, these results show that the confirmation bias likely results from a matching heuristic, such that visual codes involved in representing the search goal prioritize stimuli possessing these features.
\end{abstract}

Keywords Attention - Selective $\cdot$ Cognitive and attentional control $\cdot$ Search

As effortless as it seems, visual perception is not a passive process. The literature on visual attention is rife with examples

Jason Rajsic

jason.rajsic@mail.utoronto.ca

1 University of Toronto, Toronto, Canada of how selection processes shape what visual information reaches awareness and goes on to influence subsequent behavior (Raymond, Shapiro, \& Arnell, 1992; Simons \& Chabris, 1999; Sligte, Scholte, \& Lamme, 2008). Information selected at any given moment emerges from multiple sources of control (see Awh, Belopolsky, \& Theeuwes, 2012), with selection not always being optimal for a specific task. Although failures of attention often stem from stimulus-driven sources (Lavie \& Tsal, 1994; Theeuwes, 1992), the ability to selectively attend critical events or objects can also affected by cognitive factors, such as the number of targets one must look for (Cain, Adamo, \& Mitroff, 2013; Menneer, Cave, \& Donnelly, 2009), the specificity of a target template (Vickery, King, \& Jiang, 2005), and one's working memory capacity (Fukuda \& Vogel, 2009). A recent example of how cognitive states can influence attention is the confirmation bias in visual search (Rajsic, Wilson, \& Pratt, 2015).

Confirmation bias refers to the tendency to selectively process information in relation to a focal hypothesis (Nickerson, 1998). The bias towards confirmation is most strongly associated with Wason's research $(1960,1968)$ showing that thinkers tend not to sample information about what would not happen if a rule were true. Noting similarities in the cognitive explanations of the confirmation bias (Mynatt, Doherty, \& Dragan, 1993) and theories of visual selection (Olivers, Peters, Houtkamp, \& Roelfsema, 2011; Wolfe, Cave, \& Franzel, 1989), Rajsic et al. found that visual selection would be biased toward one of two stimulus types, depending on which type of stimulus the search was being framed as "for," even when this entailed processing more information. This result establishes, and provides a method for studying the tendency to prioritize a subset of all task-relevant information based on the mere framing of a search. As well, it highlights a commonality between reasoning and our perception of the environment; both exhibit biased information sampling. 
Indeed, confirmatory visual search patterns suggests that people may be blind, or at least slow to notice, states of the environment that they do not expect to be true under conditions of focused attention (Simons \& Chabris, 1999).

To measure whether participants were biased towards one of two possible search targets, Rajsic et al. (2015) adapted a subset search design (Bacon \& Egeth 1997; Sobel \& Cave, 2002) to include two different targets. Specifically, participants searched for a target letter that could appear in one of two colors, and were instructed to press one key if the object appeared in the first color, but to press another key if the target appeared in "another" color. We refer to the color that was shown in the instructions as the "template" and to the color that did not appear in the instructions as the "nontemplate." Indeed, confirmatory selection appeared to be the default search heuristic; search was consistently biased toward the template-colored objects even when it would have been more efficient to search through the non-template-colored objects. It is not, however, known what sorts of templates lead to such confirmatory selection. Thus, the purpose of this article is to determine when task framing will bias search towards certain stimuli over others, depending on how a search goal is phrased. In doing so, the source of this confirmation bias can be better understood.

Like many other attentional heuristics - to items held in visual working memory (Soto, Hodsoll, Rotshtein, \& Humphreys, 2008), to stimuli with learned value (Anderson, Laurent, \& Yantis, 2011), to locations with statistical structure (Zhao, Al-Aidroos, \& Turk-Browne, 2013), and to stimuli with unique visual features (Theeuwes, 1992; Franconeri \& Simons, 2003) - the confirmation bias in search appears to be an unintentional bias towards some objects by virtue of a nonperceptual property they possess. That property is their being framed as positive information in the context of a prompt, and as such, the confirmation bias in search is an attentional bias resulting from the mere framing of a search task. Rajsic et al. (2015) measured confirmation bias in search using a task where search stimuli are presented in two different colors, with the target stimulus (e.g., a $p$ among $d \mathbf{s}, q \mathrm{~s}$, and $b \mathbf{s}$ ) being equally likely to appear in either color. Orthogonally, the proportion of search stimuli of a given color varied while the total search set size was held constant. Instructions were given to report whether the target letter was a particular color or not, and given that either color may have been mentioned in the instructions for a given participant, block, or trial, selection biases towards this color must have come from these instructions. An unbiased observer would have preferentially searched the smaller set of colored stimuli; because the target appeared on every trial, the rule can be confirmed or falsified simply by having searched one color set exhaustively. If the target was not among the smaller color set, it must have been on the other color set. Instead, participants exhibited a bias toward the confirmatory color set.
What is it about the instructions that leads to selection biases? One possibility is that the instructions bias search because they present participants with a specific visual input that matches one of the stimulus colors. In their experiments, Rajsic et al. (2015) consistently instructed participants using a colored rectangle to depict the positive template. Thus, one possibility is that confirmatory searching results from simple, bottom-up intratrial priming of the confirmatory color (e.g., Theeuwes, Reimann, \& Mortier 2006).

Another possibility is that mentioning one of two possible target features in the instructions primes categorical attentional guidance processes. Guided search, for example, proposes that the selection of relevant colored stimuli in a search array depends on broadly tuned, categorical color channels (Wolfe, 2007; Wolfe et al., 1998). A categorically tuned architecture is ideal for top-down control, given that goals of a search would often begin with a linguistic code in everyday situations (e.g., saying to a friend, "That blue car looks expensive"), but especially in the context of psychology experiments where participants are instructed with written or spoken guidelines. If the confirmation bias results from a heuristic matching process between elements named in the instructions and this categorical guidance apparatus, then the confirmation bias should be observed when templates are specified only using words, not visual depictions. Experiment 1 tests this account against the possibility that confirmatory search biases are due to bottom-up priming.

If visual attention is truly attracted to confirmatory stimuli, confirmation biases should extend beyond situations in which stimuli match a particular template on a single, explicitly mentioned, homogenous visual feature. Instead, stimuli should attract attention because of their ability to verify a proposition per se, even when this proposition involves more abstract classes of stimuli. Although searching red stimuli when asked whether a target is red or not could reflect a preference to find information that would yield an affirmative answer - a true confirmation bias - it could also be due to a heuristic of relevance, such that stimulus features mentioned in the rule are heuristically deemed more important, or informative (Sperber, Cara, \& Girotto, 1995). Experiments 2, and 3 were conducted to distinguish true confirmatory search from a relevance heuristic by measuring whether biases occur when confirmatory stimuli are defined using negation (Experiment 2) and when confirmatory stimuli are visually heterogeneous (Experiment 3 ).

\section{Experiment 1}

Experiment 1 was conducted to determine whether confirmation biases in visual search are mere instances of bottom-up priming of visual features or whether they can occur when a template is described verbally. To do so, we adapted the methods and stimuli from Rajsic et al. (2015). Participants 
were instructed that, on each trial, they should evaluate whether a particular question about the display should be answered in the affirmative or negative. Specifically, all trials asked whether a particular letter was on a circle of a particular color. Instead of using a colored square to communicate the particular color, as in Rajsic et al. (2015), our study used a verbal label for each color (e.g., "red"). If participants search in a biased manner, they should preferentially search the template-matching (confirmatory) color, resulting in increased search times when the template-matching group is more numerous. If participants search in a strategic manner-ignoring confirmation bias - they should preferentially search the color with fewer circles on a trial-to-trial basis.

\section{Method}

\section{Participants}

Sixteen undergraduate students volunteered to participate for course credit. All participants provided informed consent.

\section{Stimuli}

Search displays consisted of eight letters, presented on the circumference of an imaginary circle centered on a central fixation cross. Each letter in a search display was a lowercase $p, q, b$, or $d$, approximately $2^{\circ}$ in height and $1^{\circ}$ in width, and was drawn approximately $8^{\circ}$ from fixation using Arial font drawn in black (RGB: $0,0,0)$. These letters were placed on top of small discs (approximately $1^{\circ}$ in radius) whose colors were selected from a pool of seven possible colors; purple, yellow, green, orange, pink, blue, and red (RGB values, respectively: 200, 0, 255; 200, 200, 0; 0, 255, 0; 255, 128, 0; 255, 128, 255; $50,50,255 ; 255,50,50)$, with the background set as midgray (RGB: 128, 128, 128). Before beginning a block of trials, participants were presented with instructions written on the computer monitor in the following form: "For each trial, answer this question: "Is the $x$ on a $y$ circle?" Press key 1 if yes, press key 2 if no." For a given instruction $x$ would be the target letter ( $p, b, d$, or $q$ ), $y$ would be the categorical color name, and keys 1 and 2 would refer to either the $\mathrm{Z}$ or X key, which were alternately used as either response. For example, as illustrated in Fig. 1a, participants may have been prompted to respond as to whether the $p$ was on a red circle, using the $\mathrm{Z}$ key for yes and the $\mathrm{X}$ key for no. Subsequent searches would include distractor letters on red and blue circles, with target $p$ s appearing either on a red or blue circle from trial to trial. These instructions remained on screen until participants chose to begin the corresponding block. Figure 1a depicts a sample instruction and search display (at template-matching subset size four, with a matching target color).

\section{Procedure}

One experimental session consisted of 12 blocks of 24 trials, where each block consisted of four repetitions of the six experimental conditions: Target Color (template matching or template mismatching) $\times$ Template Matching Subset Size (two, four, or six). For a given block, two of the seven possible colors were selected randomly as the two search colors to be used for the subsequent 24 trials. Two conditions were manipulated: the target color, which was template matching if it matched the color mentioned in the instructions and template mismatching if it did not, and the template-matching subset size, which could be two, four, or six stimuli. The actual target color on a given trial was equally likely to be template matching and template mismatching, regardless of template-matching subset size, and participants were informed of this overall pattern.

A given trial began with the presentation of a blank screen with a fixation cross for 2,000 ms. Following this period, the search display was presented until a response was given. After a response was entered, using either the $\mathrm{Z}$ or $\mathrm{X}$ key, written feedback about response accuracy ("Correct" or "Incorrect") was displayed in the center of the screen for 2,000 ms. After feedback offset, the next trial began.

\section{Results and discussion}

To determine whether confirmation bias occurred with bottom-up priming concerns removed, we analyzed the effect of template matching subset size and target color on median correct response times (RTs), where we expect a monotonic effect of template-matching subset size if selection is biased toward template-confirming stimuli. Two participants were excluded for having either lower than $80 \%$ accuracy or average RT more than two standard deviations from the group mean (i.e., greater than $2,890 \mathrm{~ms}$ ). Both template-matching subset size, $F(2,26)=73.46, p<.001, \eta_{\mathrm{p}}^{2}=.85$, and target color, $F(1,13)=51.51, p<.001, \eta_{\mathrm{p}}{ }^{2}=.81$ affected RT, as well as an interaction, $F(2,26)=10.60, p<.001, \eta_{\mathrm{p}}{ }^{2}=.45$. Follow-up contrasts on template-matching subset size showed a linear trend, $F(1,13)=86.78, p<.001, \eta_{\mathrm{p}}{ }^{2}=.87$, but only a marginally significant quadratic trend, $F(1,13)=3.71, p=.08, \eta_{\mathrm{p}}{ }^{2}=$ .22. Median correct RT is shown in Fig. 2. An analysis of accuracy revealed only a main effect of target color, $F(1,13)$ $=7.66, p=.016, \eta_{\mathrm{p}}^{2}=.37$, such that template-mismatching targets were reported more accurately $(M=95 \%, S E=1 \%)$ than template-matching targets $(M=92 \%, S E=1 \%)$.

To ensure that our effects were not due to speed-accuracy trade-offs, we calculated an efficiency score, mean accuracy divided by median response time, for each participant in each condition. Similarly to median correct RT, efficiency declined as template-matching subset size increased, $F(2,26)=61.52$, $p<.001, \eta_{\mathrm{p}}^{2}=.83, M_{\mathrm{SS} 2}=0.91, M_{S S 4}=0.72, M_{\mathrm{SS} 6}=0.61$. Efficiency was also lower for template-matching targets $(M=$ 


\section{a}
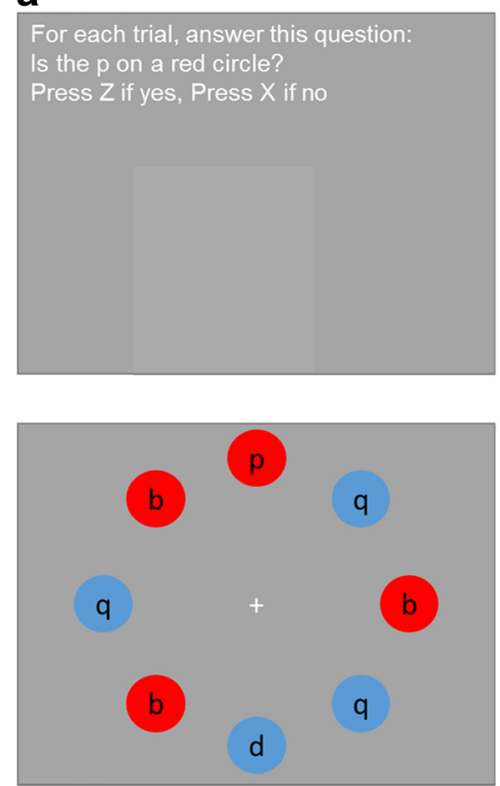

\section{b}
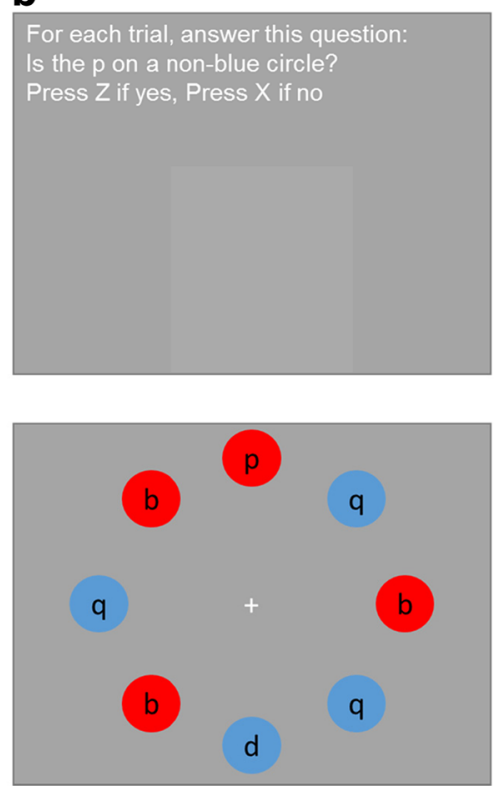

C
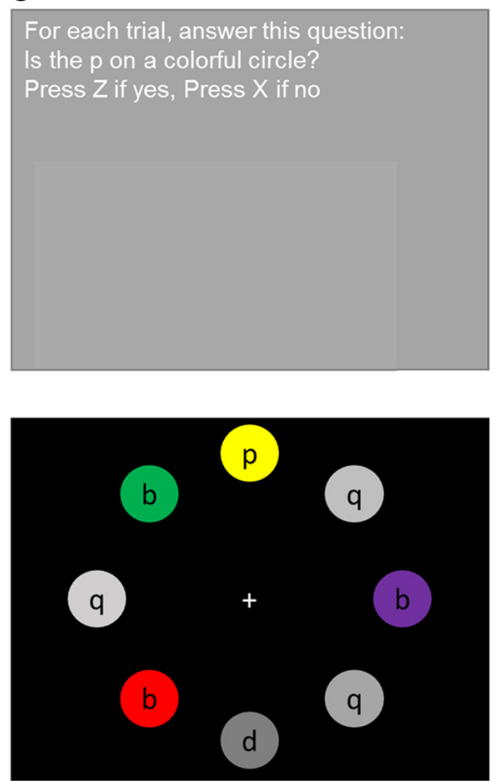

Fig. 1 A sample search instruction (upper row) and sample search array (lower low) for Experiments 1, 2, and 3 (Columns a, b, and c). Stimuli are not drawn to scale (Color figure online)

$0.80)$ than for template-mismatching targets $(M=0.70)$. Thus, the confirmatory search bias we observed was not due to a speed-accuracy trade-off.

Overall, these data show that confirmatory searching occurs even when template colors are not visually presented, but instead conveyed through language. Therefore, it is not the case that confirmatory search biases are simply due to bottom-up visual priming from instructions. Rather, confirmatory templates can be formed verbally, implying a level of nonperceptual, semantic abstraction.

\section{Experiment 2}

Experiment 1 showed that search is biased toward information that could lead to an affirmative endorsement of a visual

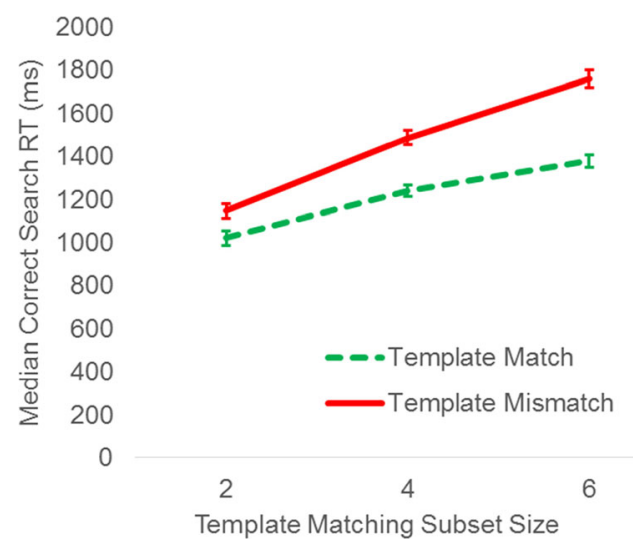

Fig. 2 Median response times in Experiment 1. Error bars in this and all other figures depict one within-subjects standard error (Cousineau, 2005) hypothesis; when search was framed as being about the presence of one target and not another, even though both targets were equally likely, stimuli matching the color of the framed color attracted attention. Critically, this occurred in the absence of any visual presentation of the target color in the instructions, leading to the conclusion that confirmation bias in search is not due to visual priming, but may derive from categorical guidance mechanisms (e.g., Wolfe et al., 1989).

In Experiment 2, we sought to determine whether the confirmatory search bias is due to a more abstract coding of relevance. In Experiment 1, all stimuli that matched a template matched by virtue of having the same feature. In research on reasoning using the Wason selection task, a number of researchers have emphasized a distinction between truly confirmatory data selection, where data are selected because they could be consistent with the proposition being evaluated, and a relevance heuristic wherein the objects or classes mentioned in the proposition being evaluated are rendered more salient (reviewed in Evans, 1998). A common technique for dissociating these two possibilities is to introduce negation in to the proposition being evaluated, so that the positive set is no longer explicitly mentioned (e.g., "If there is an A on the front of a card, there is not a 7 on the back" does not mention a particular stimulus as a true consequent). Thus, in Experiment 2 we pursued the question of whether confirmatory search patterns result from a matching bias by including blocks where one stimulus color was referred to by negation (i.e., in a block of red and blue stimuli, asking participants whether a target letter was on a "nonred" circle in lieu of "blue" circle").

\footnotetext{
${ }^{1}$ We thank Todd Horowitz for suggesting this experiment.
} 
Notably, the visual stimuli in this experiment are identical to Experiment 1. Moreover, the information provided in the prompt is equivalent. The only difference is the negative condition. Thus, the visual information and the logical information available to observers in Experiments 1, and 2 are the same. The question is whether the negative clause disrupts observers' ability to use the template to guide search. If confirmatory selection is based on the ability of stimuli to yield an affirmative response, then we should observe similar search patterns between the standard and negation search conditions. However, if selection is due to a matching bias, the negation search RT will not increase as the template-matching subset size increases.

\section{Method}

\section{Participants}

Nineteen undergraduate students were recruited for a second experiment. All participants provided informed consent and were compensated with course credit. Participants were run until the postexclusion sample size of Experiment 1 (14) was reached after using the same exclusion criteria.

\section{Stimuli and procedure}

Stimuli and procedure were identical to Experiment 1, with the following exception: Blocks were divided into two types. Standard blocks included instructions in the same format as Experiment 1, whereas in negation blocks, participants answered questions of the form "Is the $x$ on a non- $y$ circle?" These blocks were presented in a random order, determined separately for each participant. Figure $1 \mathrm{~b}$ depicts a sample negated instruction and search display (at template-matching subset size four, with a matching target color).

\section{Results and discussion}

Median correct RTs were analysed, with target color, template-matching subset size, and negation as factors. Five participants were excluded for having accuracy lower than $80 \%$ or average RT more than two standard deviations above the group mean (i.e., greater than $3,520 \mathrm{~ms}$ ). Overall, both target color, $F(1,13)=9.73, p=.008, \eta_{\mathrm{p}}{ }^{2}=.43$, and template-matching subset size, $F(1,13)=9.30, p=.001, \eta_{\mathrm{p}}{ }^{2}$ $=.42$, affected search time. Critically, negation interacted with both target color, $F(1,13)=7.66, p=.016, \eta_{\mathrm{p}}{ }^{2}=.37$, and template-matching subset size, $F(2,26)=3.93, p=.032, \eta_{\mathrm{p}}{ }^{2}=$ .23 (see Fig. 3). As such, we analyzed search performance separately for the standard and negation. Accuracy was not affected by any factors or their interaction, and so was not analysed further, $F \mathrm{~s}<1.93, p \mathrm{~s}>.17, \eta_{\mathrm{p}}^{2}<.13$.
For standard trials, target color affected correct search times, $F(1,13)=17.30, p=.001, \eta_{\mathrm{p}}^{2}=.57$, as did template-matching subset size, $F(2,26)=23.23, p<.001$, $\eta_{\mathrm{p}}{ }^{2}=.64$, accompanied by an interaction, $F(2,26)=3.51, p$ $=.045, \eta_{\mathrm{p}}^{2} \eta_{\mathrm{p}}{ }^{2}=.21$. Template-matching subset size showed significant linear, $F(1,13)=35.19, p<.001, \eta_{\mathrm{p}}{ }^{2}=.73$, and quadratic trends, $F(1,13)=7.30, p=.018, \eta_{\mathrm{p}}{ }^{2}=.36$. Follow-up paired $t$ tests, while search RT increased as template-matching subset size increased from two to four for both template-matching, $t(13)=3.60, p=.003$, and template-mismatching targets, $t(13)=8.22, p<.001$, increases from subset size four to six did not lead to a significant increase in search RT for template-matching, $t(13)=0.04, p=$ .97 , or template-mismatching targets, $t(13)=1.83, p=.09$. However, given that the search RT was faster for template-matching targets than template-mismatching targets at subset size 6 , participants showed an overall confirmatory search tendency.

For negation trials, neither factor, nor their interaction, affected search RT, $F \mathrm{~s}<1.02, p \mathrm{~s}>.37, \eta_{\mathrm{p}}{ }^{2}=.07$. At the end of each experimental session, participants reported their search strategies. Those who reported that, when shown a template-matching subset Size six display, they would choose to first inspect a Template-Mismatching Target were classified as "strategic" searchers, whereas those who reported that they would choose to first inspect a Template-Matching Target (despite the larger subset size) were classified as "confirmatory" searchers. Overall, seven participants were classified as confirmatory searchers, and seven were classified as strategic searchers. However, an analysis of negation trials showed that search strategy did not interact with template-matching subset size, $F(2,24)=0.06, p=.94, \eta_{\mathrm{p}}{ }^{2}=.005$, target color, $F(1,12)$ $=2.40, p=.15, \eta_{\mathrm{p}}^{2}=.17$, nor their combination, $F(1,12)=$ $0.04, p=.96, \eta_{\mathrm{p}}{ }^{2}=.003$. The same was true for standard trials, $F \mathrm{~s}<0.35, p \mathrm{~s}>.63$; reported search strategy did not modulate the search strategy indicated by search RT.

One reason that the negation condition may not have shown confirmatory searching is due to an asymmetry in information between these conditions. In the standard condition, the color of the implied template was mentioned in the rule, whereas in the negation condition, only the color of the implied non-template was mentioned. As such, participants may have searched in a confirmatory manner once they knew the implied template's color; that is, later in a given block. To assess this possibility, we analysed search performance for both standard and negation trials with the additional factor of block half (first vs. last). For standard trials, block half showed no main effect, $F(1,13)=3.13, p=.10, \eta_{\mathrm{p}}{ }^{2}=.19$, nor interactions, $F_{\mathrm{s}}<1.68, p \mathrm{~s}<.21, \eta_{\mathrm{p}}{ }^{2} \mathrm{~s}<.12$, with template-matching subset size or target color. On the other hand, in negation trials, template-matching subset size affected RT, $F(2,26)=3.77, p=.037, \eta_{\mathrm{p}}{ }^{2}=.23$, and accuracy, $F(2$, 26) $=4.86, p=.016, \eta_{\mathrm{p}}{ }^{2}=.27$. In the first half, search RTs 
Standard Prompt

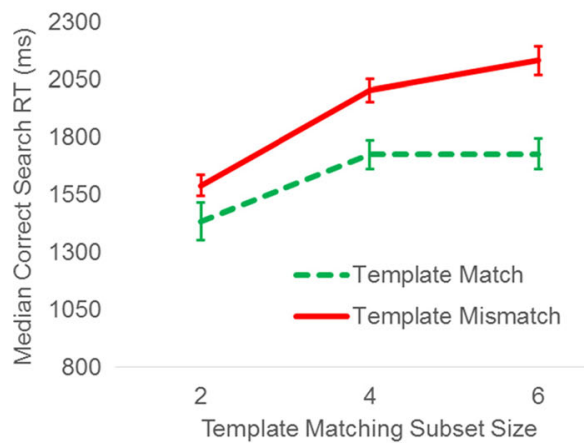

Negation Prompt
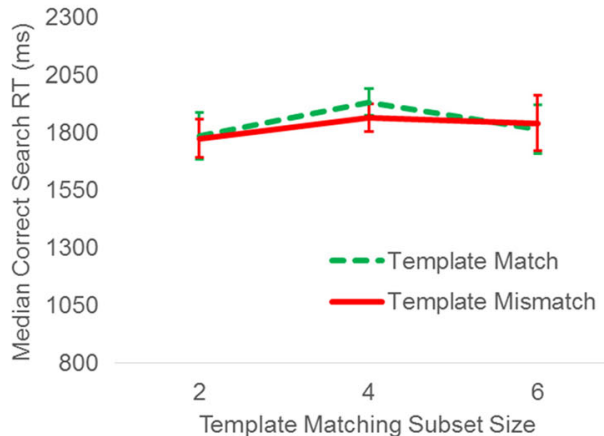

Fig. 3 Median response times Experiment 2 for standard prompts (left) and negation prompts (right)

were notably longer for matching-subset sizes four and six $(M \mathrm{~s}=1,977 \mathrm{~ms}, 1,937 \mathrm{~ms}$, respectively; SEs $=183 \mathrm{~ms}, 176$ $\mathrm{ms}$, respectively) compared to two $(M=1,779 \mathrm{~ms}, S E=151$ $\mathrm{ms})$. In the second half, however, RTs were very similar across all matching-subset sizes $\left(M_{[2,4,6]}=1,844 \mathrm{~ms}, 1,839 \mathrm{~ms}\right.$, $1,799 \mathrm{~ms}$, respectively; $S E_{[2,4,6]}=150 \mathrm{~ms}, 135 \mathrm{~ms}, 117 \mathrm{~ms}$, respectively). As such, there is a suggestion of confirmatory searching with negation instructions, but certainly it is not as clear or consistent as standard instructions.

Overall, the results of Experiment 2 show that confirmatory search biases disappear when the goals of search are framed using negation. Indeed, neither template-matching subset size nor target color affected search patterns when the target question included a negation. This suggests that no color-based selection occurred in this case. It is, however, difficult to distinguish this possibility from the alternative that search strategies differed across participants. What we can conclude is that instructions that refer to a negated feature do not reliably produce confirmatory search.

\section{Experiment 3}

The results of Experiment 2 demonstrate that visual confirmation biases do not occur when search goals are communicated using negation (i.e., when looking for a target without a particular property). Despite the search stimuli being identical across negation and standard blocks, search strategy differed markedly. However, it possible that search is biased to stimuli that are confirmatory in an abstract sense when negation is removed. Our previous demonstrations of confirmatory search have all relied on situations in which a tested proposition refer to the presence or absence of a single, visual feature, meaning that participants could create a single visual template, or expectation, in advance of a search for stimuli possessing that feature. In Experiment 3, we ask whether confirmatory search biases rely on this ability - to prepare a single visual template in advance - or whether a set of stimuli that are visually heterogenous might all attract attention solely because they could affirm a proposition. This provides a strong test of the possibility that participants select information because of its abstract ability to verify a proposition. The guidance of attention can be diluted when multiple potential target types are searched for (Menneer et al., 2009; van Moorselaar, Theeuwes, \& Olivers, 2014; but see Beck, Hollingworth, \& Luck, 2012), suggesting that a confirmatory template for a visually heterogenous set of target types is unlikely unless stimuli are able to be rapidly perceived as confirmatory, and subsequently selected.

To test for attention biases toward visually heterogenous, but confirmatory, stimuli, Experiment 3 used instructions that referred not to individual colors, but instead to the presence or absence of color (i.e., saturation). Here, we expect that visual grouping processes involved with guidance (Duncan \& Humphreys, 1989) will not contribute to salience, leaving only the categorical match between stimuli and the representation of search goals.

\section{Method}

\section{Participants}

Seventeen undergraduates volunteered to participate in Experiment 3. All participants provided informed consent and were compensated with course credit. Participants were run until the included sample size of Experiment 1 (14) was matched after performance-based exclusions, using the same criteria as Experiment 1.

\section{Stimuli and procedure}

The stimuli and procedure for Experiment 3 were identical to those of Experiment 1, with two exceptions. First, instead of using subsets of two different colors, one stimulus subset was now composed of random samples from the colors used in Experiment 1, whereas the other was composed of seven shades of gray (RGB values: 77, 77, 77; 102, 102, 102; 128, 128,$128 ; 153,153,153 ; 179,179,179 ; 204,204,204 ; 230$, 
230, 230). To ensure that all search stimuli were luminance increments relative to the background, we set the background screen color to black (RGB: 0, 0, 0).

Second, the instructions were changed such that, instead of participants answer a question about whether a target letter was on a specifically colored circle, participants were instructed in one of two ways. The question posed to participants was either "Is the $x$ on a colorful circle" or "Is the $x$ on a gray circle." Participants completed an equal number of both block types (six). Block order was again determined randomly for each participant. Figure 1c depicts a sample colorful-search instruction and search display (at template-matching subset size four, with a matching target color).

\section{Results and discussion}

Median correct RTs were again analyzed, with the additional factor of color category - that is, whether participants answered a questions about whether the target letter was on a gray circle or on a colorful circle. Three participants were excluded from analysis for either accuracy lower than $80 \%$ or average RT more than two standard deviations above the group mean (i.e., greater than $2,830 \mathrm{~ms}$ ). Overall, only target color, $F(1,13)=11.36, p=.005, \eta_{\mathrm{p}}{ }^{2}=.47$, affected correct search RT, such that trials that led to a "yes" response were overall faster $(M=1,793 \mathrm{~ms}, S E=100 \mathrm{~ms})$ than trials where a "no" response was given $(M=1,981 \mathrm{~ms}, S E=97 \mathrm{~ms}$; see Fig. 4). Critically, no effect of template-matching subset size was found, $F(2,26)=1.10, p=.38, \eta_{\mathrm{p}}^{2}=.07$, indicating participants did not select stimuli on the basis of their color category.

Furthermore, template-matching subset size did not statistically interact with color category, $F(2,26)=0.27, p=.77$, $\eta_{\mathrm{p}}{ }^{2}=.02$, target color, $F(2,26)=0.31, p=.73, \eta_{\mathrm{p}}{ }^{2}=.02$, nor their combination, $F(2,26)=2.26, p=.13, \eta_{\mathrm{p}}{ }^{2}=.15$. Finally, no factors or interactions affected search accuracy, $F \mathrm{~s}<1.70$, $p s>.20, \eta_{\mathrm{p}}^{2}<.12$.

To summarize, while a postperceptual confirmation bias was present in this task, such that affirmation of the question being evaluated was faster than rejection, we did not find evidence that stimuli were prioritized for search on the basis of their template-matching features. This result indicates that dimension-level perceptual frames do not spontaneously used to guide search.

\section{Experiment 4}

Although Experiment 3 did not reveal a confirmatory search tendency when stimuli are heterogenous, this may reflect an inability to guide attention to stimuli sharing a more abstract feature, like hue, or its absence. To determine whether the lack of guidance in Experiment 3 was due to an inability to select a heterogenous group of stimuli or due to a lack of a bias, we conducted a fourth experiment where the target letter could be in the template-matching subset or not present at all. In this situation, selecting the template-matching subset is an ideal strategy. Thus, if heterogeneously colored stimuli can be selectively searched when selection would improve performance, search times will increase in proportion to the size of the template-matching subset.

\section{Method}

\section{Participants}

Fourteen participants, none of whom participated in any of the previous experiments, participated in Experiment 4. All of the participants were enrolled in a first-year undergraduate Psychology course at the University of Toronto, and were compensated with course credit for their participation. Participants all gave informed consent before participating.

\section{Stimuli and procedure}

Stimuli and procedure were identical to those of Experiment 3, with two exceptions. First, target letters appeared on one of the template-matching search stimuli on half the trials, but on the other half of the trials all letter stimuli were nontargets. Second, the instructions at the beginning of each block were changed to reflect this modification. The prompt for Experiment 4 was, "For each trial, answer this question: Is the $<$ target letter $>$ on a $<$ colorful/gray $>$ circle? Press $<$ key $1>$ if yes, Press $<$ key $2>$ if no," where angular brackets depict variable contents (i.e., the target letter could be $p, d, b$, or $q$ ).

\section{Results}

One participants was excluded from analysis for having an average RT greater than two standard deviations from the group mean $(3,038 \mathrm{~ms})$. Median search RTs can be seen in Fig. 5. Template-matching subset size, $F(2,12)=127.32, p<$ $.001, \eta_{\mathrm{p}}{ }^{2}=.91$, target presence, $F(2,12)=172.16, p<.001$, $\eta_{\mathrm{p}}{ }^{2}=.94$, and color category, $F(2,24)=14.37, p=.003, \eta_{\mathrm{p}}{ }^{2}=$ .55 , all affected search RTs, with an interaction between target presence and template-matching subset size, $F(2,12)=35.02$, $p<.001, \eta_{\mathrm{p}}{ }^{2}=.74$. As can be seen in Fig. 5, for both color categories, search slopes were linear, with target-absent searches being notably slower. A linear contrast for template-matching subset size, $F(1,12)=151.52, p<.001$, $\eta_{\mathrm{p}}{ }^{2}=.93$, with no quadratic contrast, $F(1,12)=0.07, p=.80$, $\eta_{\mathrm{p}}{ }^{2}=.006$, showed that searches were restricted to appropriate category set. Searches were faster when the target was present $\left(M_{\text {present }}=1,772 \mathrm{~ms}, S E_{\text {present }}=88 \mathrm{~ms}, M_{\text {absent }}=1,335 \mathrm{~ms}\right.$, $\left.S E_{\text {absent }}=63 \mathrm{~ms}\right)$. An analysis of accuracy also showed higher accuracy for target-absent $(M=94.8 \%, S E=1.2 \%)$ than 

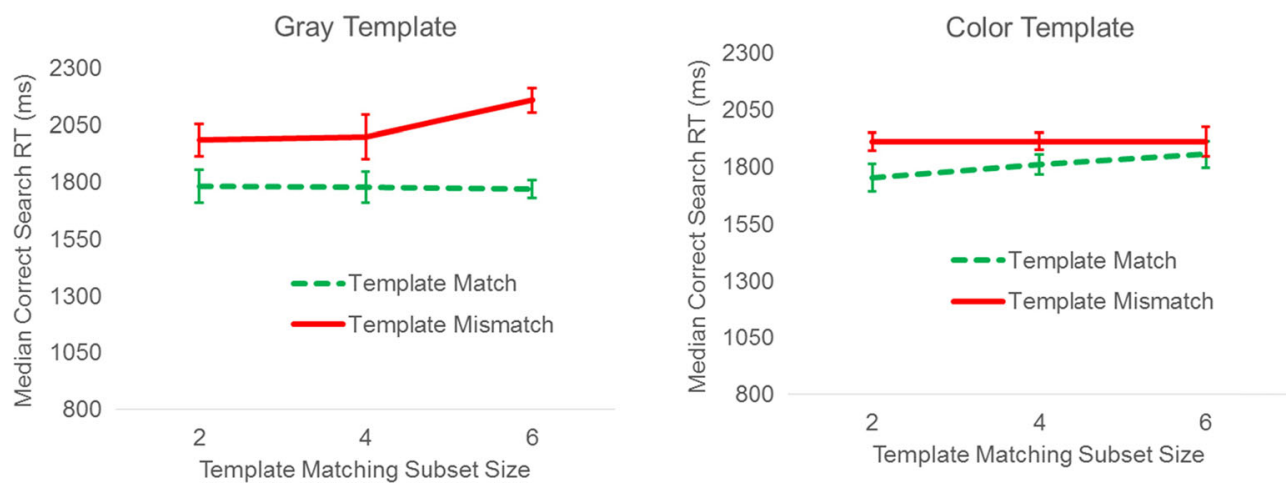

Fig. 4 Median correct response times in Experiment 3 for gray templates (left) and colorful templates (right)

target-present $(M=90.1 \%, S E=2.0 \%)$ searches, suggesting that miss errors were more common than false alarms, $F(1,12)$ $=12.34, p=.004, \eta_{\mathrm{p}}^{2}=.51$. Target-matching subset size, also affected accuracy, $F(2,12)=6.46, p=.006, \eta_{\mathrm{p}}^{2}=.35$, such that accuracy declined as subset size increased $\left(M_{[2,4,6]}=93.7\right.$, $92.9,90.8 \%$, respectively; $S E_{[2,4,6]}=1.6,1.6,1.6 \%$, respectively), suggesting that both misses and false alarms occurred more often when more stimuli matched the search template, a trend that was present in the confirmatory searches found in Experiments 1, and 2. Overall, however, these data show that searches can be guided towards a heterogeneous color category (the presence or absence of hue), but the confirmation bias does not occur in this situation.

\section{General discussion}

The goal of this study was to determine the level of representation at which biases in attention induced by the framing of a search goal occur. Previous research has shown that, in a search for two possible target conjunctions, simply phrasing the instructions such that one target is the absence of another target will lead to preferential selection of the latter target possibility (Rajsic et al., 2015). However, these results are attributable to a range of possible representational sources, ranging from simple visual priming to an abstract, logical target code. These results demonstrate that confirmatory biases, as they exist in visual search, occur when one possible

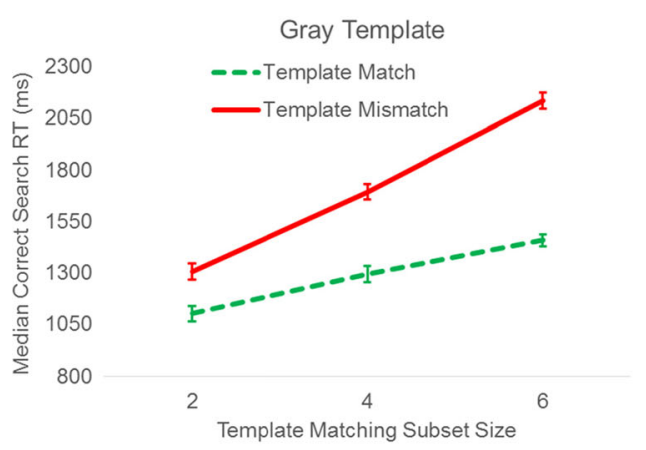

target type is defined by the presence of a visual feature (i.e., the color "red"), but not when positive templates consist of a set of visual features (i.e., any colored stimulus) or the absence of a visual feature (i.e., not red). This suggests that confirmation bias results from a sort of conceptual priming, such that propositions that can be translated into a single, categorical visual template can produce search biases for instances of this visual template. This is consistent with the finding that the presentation of verbal labels of objects speeds their entry in to awareness (Lupyan \& Ward, 2013) and orients attention (Spivey, Tyler, Eberhard, \& Tanenhaus, 2001), as well as findings that visually specific templates guide attention better than more abstract templates (Hout \& Goldinger, 2014; Maxfield \& Zelinsky 2012; Vickery et al., 2005). Furthermore, it is consistent with findings that negative information tends not to guide attention in visual search (Beck \& Hollingworth, 2015; Becker, Hemsteger, \& Peltier, 2016; Moher \& Egeth, 2012).

Given the contrast between the results of Experiment 4, which demonstrate an ability to attend to a heterogeneous subset, and the results of Experiment 3, which show no bias toward heterogeneous subsets due to the task framing, we must emphasize that the confirmation bias in visual search appears to be just that: a bias. Following Rajsic et al. (2015), we interpret data from these experiments as indicating the presence of cognitive heuristics in search that can, in certain circumstances, be overcome. Indeed, we have found that searches in which information obtained more slowly show a reduced confirmation bias (Rajsic, Wilson, \&

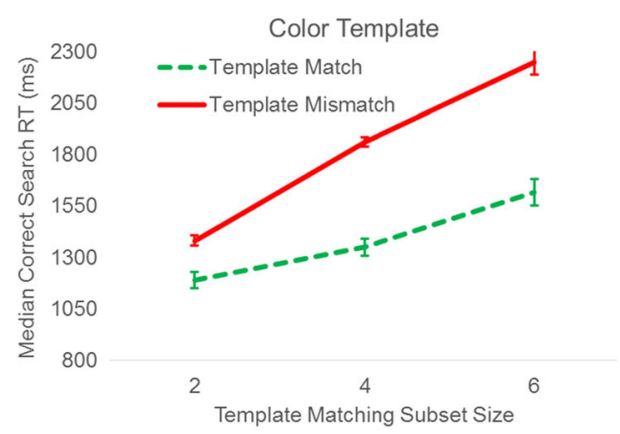

Fig. 5 Median search times for Experiment 4. Error bars reflect 1 within-subjects standard deviation of the mean 
Pratt, 2016). Furthermore, Walenchok, Goldinger, and Hout (2016) have shown that confirmatory searching patterns are reversed when template-matching targets are less common than template-mismatching targets, suggesting search efficiency takes precedent over cognitive framing. Overall, the available evidence suggests that cognitive economy is an important factor in the presence of cognitive heuristics in attention (see also Irons \& Leber, 2016).

Another important conclusion of this study is that merely framing one class of stimuli as positive instances of a hypothesis does not guarantee that they will be prioritized. What appears to be necessary for this bias to emerge is for positive instances to share a common visual feature and for that feature to be explicitly stated in advance. As such, we speculate that the mechanism underlying this bias may be the visual representations that are constructed to encode and store the question being evaluated. This is consistent with the notion that attention is often involuntarily driven to stimuli with features that match information held in visual working memory (Olivers, 2009; Soto et al., 2008). In Experiments 2, and 3, because targets were defined by the absence of a feature, or by a visually heterogenous set of features, we suspect that the search instructions could not be stored as a visual code. We note, however, that in Experiment 3, we did observe an overall RT cost for template-mismatching targets, suggesting an additional, postperceptual confirmation bias.

The finding that confirmatory search exists only for nonnegative templates is consistent with research on confirmation bias using Wason's selection task (Evans, 1998; Evans \& Lynch, 1973; Wason, 1968). Although participants often neglect to select the not- $q$ card in their evaluation of an arbitrary rule (i.e., to use modus tollens), when participants evaluate the expression "if $p$ then not- $q$," their selection of the negated consequent (in this case, simply $q$ ) improves. Indeed, negation reduces card selections for both antecedent cases and consequent cases. These findings are consistent with the notion that evaluation performance in the standard task is a mixture of tendencies toward logical evaluation and tendencies, or heuristics, to select those cards with features that are mentioned by the rule (i.e., the $p$ and $q$ cards). Most theories of the matching bias explain it by appealing some sort of relevance heuristic; at the first stage of reasoning, information must be sorted by its relevance to the evaluation of a proposition (Sperber et al., 1995). Stimuli that possess features contained in the to-be-evaluated proposition are rapidly seen as relevant, whereas stimuli that may be relevant, but are not mentioned in the proposition (i.e., a false consequent when evaluating an "if $p$ then $q$ " proposition) must be recognized as relevant by mentally unpacking the proposition's implications. In this light, the visual confirmation bias does seem to be an instance of a matching bias heuristic, which is consistent with our previous work showing that it persists despite instructions to attend the smaller subset (Rajsic et al., 2015). Research on the matching bias has uncovered one salient limitation, however: the use of realistic materials and scenarios (Griggs \& Cox, 1983; Oaksford \& Stenning, 1992). In such situations, the richer knowledge base available to guide information selection and store the proposition in memory seems to reduce the effect of matching biases in data selection. As such, future research on the confirmation bias in search ought to consider using realistic materials and prompts to assess whether the matching heuristic will still apply and lead to confirmatory search patterns, especially given the ability of object category knowledge to guide attention (Maxfield \& Zelinsky, 2012; Yu, Maxfield, and Zelinsky 2016).

Acknowledgments This research was supported by a Natural Sciences and Engineering Research Council of Canada Discovery to Jay Pratt (194537) and an NSERC PGS-D Scholarship to Jason Rajsic. We would like to thank Nafisa Bhuiyan for her help with data collection.

\section{References}

Anderson, B. A., Laurent, P. A., \& Yantis, S. (2011). Value-driven attentional capture. Proceedings of the National Academy of Sciences, 108(25), 10367-10371.

Awh, E., Belopolsky, A. V., \& Theeuwes, J. (2012). Top-down versus bottom-up attentional control: A failed theoretical dichotomy. Trends in Cognitive Sciences, 16(8), 437-443.

Bacon, W. F., \& Egeth, H. E. (1997). Goal-directed guidance of attention: Evidence from conjunctive visual search. Journal of Experimental Psychology: Human Perception \& Performance, 23(4), 948-961.

Beck, V. M., \& Hollingworth, A. (2015). Evidence for negative feature guidance in visual search is explained by spatial recoding. Journal of Experimental Psychology: Human Perception and Performance, 1(5), 1190-1196. doi:10.1037/xhp0000109

Becker, M. W., Hemsteger, S., \& Peltier, C. (2016). No templates for rejection: A failure to configure attention to ignore task-irrelevant features. Visual Cognition, 1-18. doi:10.1080/13506285.2016.1149532

Cain, M. S., Adamo, S. H., \& Mitroff, S. R. (2013). A taxonomy of errors in multiple-target visual search. Visual Cognition, 21(7), 899-921.

Cousineau, D. (2005). Confidence intervals in within-subject designs: A simpler solution to Loftus and Masson's method. Tutorials in Quantitative Methods for Psychology, 1(1), 42-45.

Duncan, J., \& Humphreys, G. W. (1989). Visual search and stimulus similarity. Psychological review, 96(3), 433-458.

Evans, J. S. B. (1998). Matching bias in conditional reasoning: Do we understand it after 25 years? Thinking \& Reasoning, 4(1), 45-110.

Evans, J. S. B. T., \& Lynch, J. S. (1973). Matching bias in the selection task. British Journal of Psychology, 64(3), 391-397.

Franconeri, S. L., \& Simons, D. J. (2003). Moving and looming stimuli capture attention. Perception \& Psychophysics, 65(7), 999-1010.

Fukuda, K., \& Vogel, E. K. (2009). Human variation in overriding attentional capture. The Journal of Neuroscience, 29(27), 8726-8733.

Griggs, R. A., \& Cox, J. R. (1983). The effects of problem content and negation on Wason's selection task. Quarterly Journal of Experimental Psychology, 35(3), 519-533.

Hout, M. C., \& Goldinger, S. D. (2014). Target templates: The precision of mental representations affects attentional guidance and decisionmaking in visual search. Attention, Perception \& Psychophysics, 77(1), 128-149. 
Irons, J. L., \& Leber, A. B. (2016). Choosing attentional control settings in a dynamically changing environment. Attention, Perception \& Psychophysics, 78(7), 2031-2048.

Lavie, N., \& Tsal, Y. (1994). Perceptual Load as a major determinant of the locus of selection in visual attention. Perception \& Psychophysics, 56(2), 183-197.

Lupyan, G., \& Ward, E. J. (2013). Language can boost otherwise unseen objects into visual awareness. Proceedings of the National Academy of Sciences, 110(35), 14196-14201.

Maxfield, J. T., \& Zelinsky, G. J. (2012). Searching through the hierarchy: How level of target categorization affects visual search. Visual Cognition, 20(10), 1153-1163.

Menneer, T., Cave, K. R., \& Donnelly, N. (2009). The cost of search for multiple targets: Effects of practice and target similarity. Journal of Experimental Psychology: Applied, 15(2), 125.

Moher, J., \& Egeth, H. E. (2012). The ignoring paradox: Cueing distractor features leads first to selection, then to inhibition of tobe-ignored items. Attention, Perception \& Psychophysics, 74(8), $1590-1605$

Mynatt, C. R., Doherty, M. E., \& Dragan, W. (1993). Information relevance, working memory, and the consideration of alternatives. The Quarterly Journal of Experimental Psychology, 46(4), 759-778.

Nickerson, R. S. (1998). Confirmation bias: A ubiquitous phenomenon in many guises. Review of General Psychology, 2(2), 175-220.

Oaksford, M., \& Stenning, K. (1992). Reasoning with conditionals containing negated constituents. Journal of Experimental Psychology: Learning, Memory, and Cognition, 18(4), 835.

Olivers, C. N. (2009). What drives memory-driven attentional capture? The effects of memory type, display type, and search type. Journal of Experimental Psychology: Human Perception and Performance, 35(5), 1275.

Olivers, C. N., Peters, J., Houtkamp, R., \& Roelfsema, P. R. (2011). Different states in visual working memory: When it guides attention and when it does not. Trends in Cognitive Sciences, 15(7), 327-334.

Rajsic, J., Wilson, D. E., \& Pratt, J. (2015). Confirmation bias in visual search. Journal of Experimental Psychology: Human Perception and Performance, 41(5), 1353-1364.

Rajsic, J., Wilson, D. E., \& Pratt, J. (2016). The price of information: Inspection costs reduce the confirmation bias in visual search. The Quarterly Journal of Experimental Psychology.

Raymond, J. E., Shapiro, K. L., \& Arnell, K. M. (1992). Temporary suppression of visual processing in an RSVP task: An attentional blink? Journal of Experimental Psychology: Human Perception and Performance, 18(3), 849.

Simons, D. J., \& Chabris, C. F. (1999). Gorillas in our midst: Sustained inattentional blindness for dynamic events. Perception, 28(9), 1059-1074.
Sligte, I. G., Scholte, H. S., \& Lamme, V. A. (2008). Are there multiple visual short-term memory stores? PLoS ONE, 3(2), e1699.

Sobel, K. V., \& Cave, K. R. (2002). Roles of salience and strategy in conjunction search. Journal of Experimental Psychology: Human Perception \& Psychophysics, 28(5), 1055-1070.

Soto, D., Hodsoll, J., Rotshtein, P., \& Humphreys, G. W. (2008). Automatic guidance of attention from working memory. Trends in Cognitive Sciences, 12(9), 342-348.

Sperber, D., Cara, F., \& Girotto, V. (1995). Relevance theory explains the selection task. Cognition, 57(1), 31-95.

Spivey, M. J., Tyler, M. J., Eberhard, K. M., \& Tanenhaus, M. K. (2001). Linguistically mediated visual search. Psychological Science, 12(4), 282-286.

Theeuwes, J. (1992). Perceptual selectivity for color and form. Perception \& Psychophysics, 51(6), 599-606.

Theeuwes, J., Reimann, B., \& Mortier, K. (2006). Visual search for featural singletons: No top-down modulation, only bottom-up priming. Visual Cognition, 14(4/8), 466-489.

van Moorselaar, D., Theeuwes, J., \& Olivers, C. N. L. (2014). In competition for the attentional template: Can multiple items within visual working memory guide attention? Journal of Experimental Psychology: Human Perception and Performance, 40(4), 1450 1464.

Vickery, T. J., King, L. W., \& Jiang, Y. (2005). Setting up the target template in visual search. Journal of Vision, 5(1), 8-8.

Walenchok, S., Goldinger, S., \& Hout, M. (2016). Examining confirmatory search strategies in visual search: People are more flexible than you think. Journal of Vision, 16, 989.

Wason, P. C. (1960). On the failure to eliminate hypotheses in a conceptual task. Quarterly Journal of Experimental Psychology, 12(3), 129-140.

Wason, P. C. (1968). Reasoning about a rule. Quarterly Journal of Experimental Psychology, 20(3), 273-281.

Wolfe, J. M. (2007). Guided search 4.0. In W. D. Gray (Ed.), Integrated models of cognitive systems (pp. 99-119). Oxford: Oxford University Press.

Wolfe, J. M., Cave, K. R., \& Franzel, S. L. (1989). Guided search: An alternative to the feature integration model for visual search. Journal of Experimental Psychology: Human Perception and Performance, 15(3), 419.

Yu, C. P., Maxfield, J. T., \& Zelinsky, G. J. (2016). Searching for category-consistent features: A computational approach to understanding visual category representation. Psychological Science, 27(6), 870-884

Zhao, J., Al-Aidroos, N., \& Turk-Browne, N. B. (2013). Attention is spontaneously biased toward regularities. Psychological Science, 24(5), 667-77. doi:10.1177/0956797612460407 